\title{
Subcomponents of Vitamine B Complex Regulate the Growth and Development of Human Brain Derived Cells
}

\author{
K.E. Danielyan* \\ H. Buniatian Institute of Biochemistry, NAS, Yerevan, Armenia \\ *Corresponding author: kristine_danielyan@biochem.sci.am
}

Received January 11, 2013; Revised February 21, 2013; Accepted March 22, 2013

\begin{abstract}
The work is focused on the role of single components of Vitamin B complex: pyridoxine, riboflavin, thiamine, and nicotinamide in the processes of growth and development of the human brain derived cells. We, also, have assayed the activity of Xanthine Oxidase in the presence of above mentioned subcomponents to delineate the possible mechanism of their action. Results indicate that the all components of Vitamin B complex might be responsible for cells' growth, maturation, proliferation. During early period of the cells' growth the most important components were thiamine and Pyridoxine, initiating cells' proliferation (number of the cells in one field: 2556, $17 \pm 355$, 68, 2179,0 $\pm 223,55$, resp) vs control $(1562,94 \pm 146,45)$, whereas during the late stages of maturation the most important components responsible for differentiation, were riboflavin and nicotinamide (3774.77 \pm 188.41 , $3558.82 \pm 152.90$ resp. vs control $2905 \pm 263.75 ; \mathrm{p}<0.035$ ). In comparison with the all other subcomponents of Vitamin B complex only in pyridoxine containing samples, XO activity was specifically inhibited by allopurinol

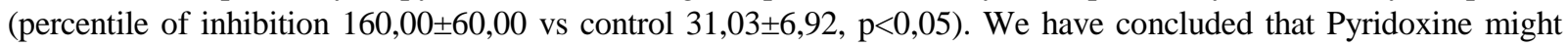
interact with XO and regulate its activity. All components of Vitamin B complex are able to initiate cells development and growth; however, they are supposed to be selectively utilized in time dependent manner to guarantee the highest efficiency of the cells development, maturation and proliferation
\end{abstract}

Keywords: subcomponents of Vitamin B, human brain, neuronal cell culture, xanthine oxidase

\section{Introduction}

The fact that thiamine deficiency (TD) is a model of chronic impairment of oxidative metabolism leading to neurodegeneration and induction of the oxidative stress and death in neurons, but not in astrocytes, micriglia or brain endothelial cells, was reported in the literature [13]. Moreover, thiamine deficiency during pregnancy leads to cerebellar neuronal death in rat offspring. The other group of the scientists reported that treatment with the vitamin oxythiamine promoted survival of hippocampal neurons, while the antagonist of oxythiamine prevented this effect [4].

The other subcomponents of Vitamin B complexpyridoxine and its derived cofactor, Pyridoxal Phosphate (PLP) were investigated. Their addition into the primary cultured neurons of various brain regions from fetal rat brain significantly promoted the neuronal survival in high cell density culture (10(5) cells/cm(2), but showed no positive effects in low density cell culture (5 x 10(3) cells/cm2). This neurotrophic effect of PLP was remarkably suppressed by picrotoxin and Ifenprodil. Aminooxyacetic acid (AOAA), an inhibitor of PLP dependent enzymes, caused significant neuronal loss, and largely counteracted the neurotrophic effect of PLP.
It is presumed that Vitamin B6 afforded the survivalpromoting activities of cultured neurons by virtue of its crucial coenzymatic actions in the biosynthesis of putative neurotransmitters [5].

Nicotinamide, next subcomponent of Vitamin B complex, a precursor of NAD, is able to block the apoptosis induced by MPTP. Moreover, nicotinamide also quenches some of the radicals formed by Xanthine Oxidase (XO) [10].

$\mathrm{XO}$ as well as the (XDH) Xanthine Dehydrogenase are two enzymes responsible for the last steps of purines metabolism, hydroxylation of a wide variety pyrimidines.

Xanthine oxidoreductase (XOR) enzyme has been isolated from a wide range of organisms, from bacteria to man. The mammalian enzymes, which catalyze the hydroxylation of hypoxanthine and xanthine, the last two steps in the formation of urate, are synthesized as the dehydrogenase form of XOR and exist mostly as such in the cell but can be readily converted to the oxidase form by oxidation of sulfhydryl residues or by proteolysis [11]. All of these proteins have similar molecular weights and composition of redox centers [6]. XDH shows a preference for NAD1 reduction at the flavin adenine dinucleotide (FAD) reaction site, whereas XO fails to react with NAD1 and exclusively uses dioxygen as its substrate, leading to the formation of superoxide anion and hydrogen peroxide [6]. 
It was shown that thiamine at concentrations to $10^{2} \mathrm{M}$ had no effect on the xanthine-xanthine oxidase-mediated reduction of ferricytochrome $\mathrm{c}$ in neutrophils and lymphocytes [18].

Rat ventral prostate xanthine oxidase-mediated metabolism of acetaldehyde to acetyl radical might be inhibited not only by allopurinol, oxypurinol, diphenyleneiodonium chloride, ellagic acid but also by folate [1].

The involvement of riboflavin-mediated formation of free radicals/reactive oxygen species and subsequent neurite degeneration in in vitro-assays of neuronal regeneration or neuronal cell cultures was reported by the German group of authors [8]. The enzymes catalase and superoxide dismutase and the antioxidant agent selenium showed some protection against hydrazine neurotoxicity, suggesting an involvement of the generation of reactive oxygen species in the pathogenesis of isoniazid, responsible for the disturbance of Vitamin B6 metabolism, neuropathy [15].

Thus, there are number of articles indicating the role of single Vitamin B complex ingredients influence on the neuronal cells culture development. This influence is mediated not only by the classically known mechanism of the involvement of these components into the processes of cell growth and development, but also via the initiation of ROS generation and even influence on XO activity.

The work described in this article is focused on the role of single components of Vitamin B complex: pyridoxine, riboflavin, thiamine, nicotinamide in the processes of cell growth and development. We have assayed also the activity of $\mathrm{XO}$ in the presence of above mentioned subcomponents to delineate the involvement of this enzyme and its ability to form ROS in the physiological processes of cell growth in vitro.

\section{Materials and Methods}

\subsection{Cell Culturing}

All procedures with the utility of biomaterials were carried out in accordance with the "Declaration of Helsinki”. Human embryos were obtained from elective abortions with the informed consent of the women seeking abortion. Moreover, as a biomaterial there were used only fetuses with the ages of formation not more than 12 weeks [14].

Brains of the human embryos (E90) were withdrawn, and placed in Neurobasal medium (NB, prenatal, Gibco Life Technologies), containing $0.05 \%$ bovine serum albumin (BSA). The tissue was isolated and incubated at $37^{\circ} \mathrm{C}$ for $20 \mathrm{~min}$ in NB containing $0.05 \%$ BSA, $0.15 \%$ Trypsin. Tissue was resuspended in fresh NB and mechanically disintegrated using a Pasteur pipette. The supernatant was discarded and the cell suspension resuspended in NB medium containing 1\% BSA. This procedure was repeated 3 times. Human brain cells were collected $(1,000 \mathrm{rpm}, 10 \mathrm{~min})$, washed and cultured at $37^{\circ} \mathrm{C}$, $5 \% \mathrm{CO}_{2}$ in $35 \mathrm{~mm}$ Petri dishes pre-coated with poly-Llysine (Sigma), containing $0.09 \% \mathrm{Na}_{2} \mathrm{HPO}_{4}, 1 \%$ glucose, $0.4 \% \mathrm{KCl}, 0.06 \% \mathrm{KH}_{2} \mathrm{PO}_{4}, 0.4 \% \mathrm{MgSO}_{4} \times 7 \mathrm{H}_{2} \mathrm{O}$ and
$0.001 \%$ gentamicin sulfate. A day later the medium was replaced by NB containing 2\% B27-supplement (Gibco) and the cells' number was calculated on days second and $12^{\text {th }}[9]$.

\subsection{Trypan Blue Staining}

Cell suspension was prepared in BSS (Hank's Balanced Salt Solution, Product No. H9269, Sigma). After, $0.5 \mathrm{ml}$ of $0.1 \%$ Trypan Blue solution was transferred to a test tube and added $0.3 \mathrm{ml}$ of BSS to $0.2 \mathrm{ml}$ of the cell suspension (dilution factor $=5$ ) and mixed thoroughly. We have allowed the cell suspension-Trypan Blue mixture to stand at least 5 minutes. Further, a small amount of the Trypan Blue-cell suspension mixture was transferred to both chambers of the hemacytometer. Nonviable cells stained blue. We have kept a separate count of viable and nonviable cells. Since $1 \mathrm{~cm}^{3}$ of the chamber is approximately $1 \mathrm{ml}$, the subsequent cell concentration/ml (and total cell number) was detected accordingly [17].

\subsection{Microscopy}

Polarizing microscope Biolar PI (PZO,Warsaw,Poland; magnification $1.25 \times 40$ or $1.25 \times 20$ ) was used. Number of the cells were calculated by utilizing of Pixcavator program.

\subsection{Xanthine Oxidase Activity Estimation by the Determination of the Uric Acid Quantity in the Brain Tissue}

Xanthine as well as allopurinol was incubated with the biological solution for one hour at $37^{\circ} \mathrm{C}$, after the Specol 2000 estimated all the absorption under 660nm of the wavelength.

\subsection{Homogenization of the Organ's Tissue}

For $100 \mathrm{ml}$ of the buffer, we used $0.87 \mathrm{~g}$ of the $\mathrm{NaCl}$, $0.06 \mathrm{~g}$ of $\mathrm{KH}_{2} \mathrm{PO}_{4}, 0.09 \mathrm{~g} \mathrm{Na} \mathrm{HPO}_{4}, 5 \mathrm{mM} \mathrm{MgCl}, 0.1 \mathrm{M}$ Tris aminomethane, $1 \mathrm{ml}$ of Triton $\mathrm{X}$ 100, 200ug of Tripsin inhibitors, as well as $0.001 \mathrm{M} \mathrm{KNaC} \mathrm{H}_{4} \mathrm{O} 6 \times 4 \mathrm{H}_{2} \mathrm{O}$. Glass-glass homogenization was performed for 20 minutes. The mixture was centrifuged at $\mathrm{G}=8000$ for 20 minutes. Supernatant was used for the experiments.

\subsection{Detection and Quantification of the Proteins by Bradford}

Coomassie Brilliant Blue G-250 (100mg) was dissolved in $50 \mathrm{ml} 95 \%$ ethanol, $100 \mathrm{ml} 85 \%(\mathrm{w} / \mathrm{v})$ phosphoric acid, diluted to 1 liter, when the dye has completely dissolved, filtered through Whatman \#1. The duration of the reaction between the Coomassie Brilliant Blue solution and the sample was 20 minutes. After all, using the Specol 2000 (Poland) and wavelength equal to $590 \mathrm{~nm}$, we have determined the concentration of the total proteins in the biological solution [7].

\subsection{Statistics}

In our calculations we have used t-test (student) for pair comparison as well as ONE-WAY-ANOVA for the 
calculation of the significance of the comparable all groups. The results were considered statistically significant when p was lower or equal to 0.05 .

\section{Results and Discussion}

\subsection{Growth of the Cells and Development over 4 and 12 Days}

In the first set of the experiments we have determined the growth of the cells as well as their death in the presence of single components of Vitamin B complex: nicotinamide, ribiflavine, pyridoxine and thiamine $(1668,50 \pm 189,51, \quad 1738,33 \pm 196,60 ; \quad 2556,17 \pm 355,68$, $2179,00 \pm 223,55$, resp.). As a positive control in these experimental series, it was used bFGF. It is clear from the experiments that in comparison with the 2 types of negative controls: number of the cells on the $1^{\text {st }}$ $(820,14 \pm 50,07)$ and 4 th $(1562,94 \pm 146,45)$ days, as well as positive control- growth of the cells in the presence of bFGF $(2131,08 \pm 144,59)$; the most effective component of Vitamin B complex was pyridoxine and thiamine. In comparison with the nicotinamide and riboflavin, these two components vividly have increased the growth of the cells even on the day 4th, Figure 1.

Thiamine, in accordance to the literature data [3], is protective for the neuronal cell culture, derived from the hippocampus. The results of the authors are in agreement with ours [3]. However, it is necessary to mention that the authors were working with the cell culture during the first 72 hours and were not monitoring the influence and dose dependent influence of this compounds during the late stages of development.

We have been trying to culture cells not only in Neurobasal/B27 (Invitrogen, Gibco) but also in HBSS. However, our results pointed on the detrimental effect of the HBSS in comparison with Neurobasal /B27 $(1392,25 \pm 55,01)$.

We have planned to examine the dynamic of the brain cells growth and maturation during the entire period of the cell culturing. Thus, the number of the cells was calculated not only on the $4^{\text {th }}$ but also on $12^{\text {th }}$ days. The results were surprising: in contrast to the first period of the cells growth and development, during the second period of time, as it is clear from the Figure 2, the most effective compound for the development and growth of the cells were not thiamine (3154.80 \pm 196.96) and Pyridoxine

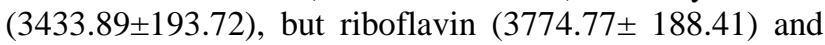
nicotinamide (3558.82 \pm 152.90$)$. In this case the numbers were significantly more higher than in the control group (2905,0 $\pm 263.75 ; \quad \mathrm{p}<0.035), \quad$ bFGF $\quad(3158.04 \pm 313.09)$ treated group and in the group, where the cells were

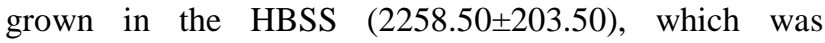
considered as a blank control group.

\subsection{Quantification of Death Cells in the Culture}

As it is clear from the Figure 3, the lowest quantity of the dead cells and cell bodies in the field was registered in the control and the other groups. The statistically significant results for comparison of the cells and cell bodies was registered for control group vs the all other groups (embryonic bodies: control- 1557.44 \pm 127.47 , nicotinamide-2692.32 \pm 185.48 , riboflavin-3522.41 \pm 119.63 , pyridoxine- 4005.30 \pm 91.02 , thiamine-3477.00 \pm 322.63 ; small cells fractions: control-213.83 \pm 15.27 , nicotinamide$281.68 \pm 5.84$, riboflavin- $299.23 \pm 7.85$, pyridoxine$307.20 \pm 5.06$, thiamine-296.40 $\pm 4.75, \mathrm{p}<0,001)$. $\mathrm{P}$ value was equal to and lower from 0.05 between nicotinamide and ribovlavin as well as nicotinamide and pyridoxine.

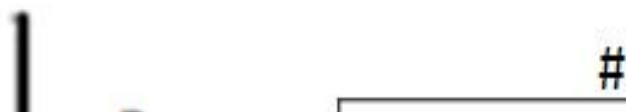

$\#$

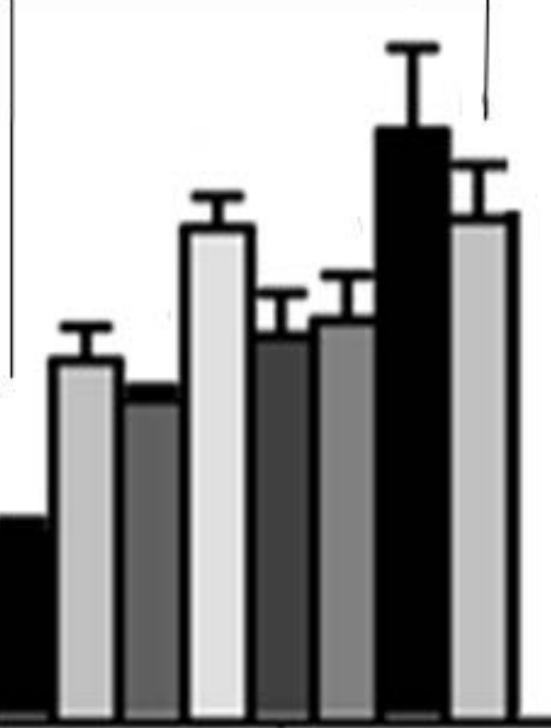

Day 1

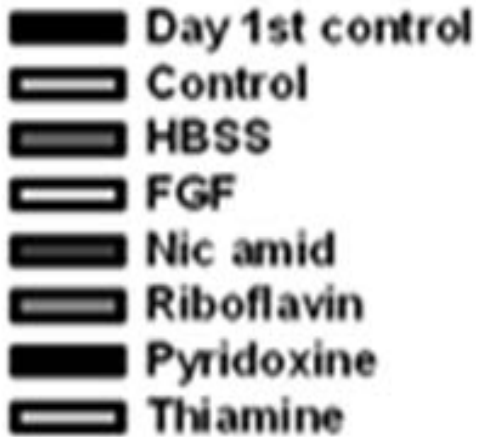


Pictures are taken on days 1st and 4th. Polarizing microscope Bipolar PI (PZO,Warsaw,Poland; magnification 60x1.25x40) was used. The cells were grown on the glass in the Petry dishes, covered with the Poly-L-Lysine. The Neurobasal/B27 containing bFGF , nicotinamide , rboflavin , pyridoxin , thiamine or low concentration of Vitamin B complex was used as cell media. The number of the cells in the field was counted using the Pixcavator program, allowing to calculate authomatically the size as well as the number of the cells. T-student test t was used to calculate the statistical significance of the results. P2, P4, P5 were higher than 0.05 , whereas P1 between control from day 1 st and control from day 4th were $<0.05$. P3, P6, P7, P8 were significantly higher in comparison with the control and lower than 0.04

Figure 1. The influence of the single components of Vitamin B complex on the growth and development of the human embryonic brain cells. A. Presented are the pictures of the brain cells.

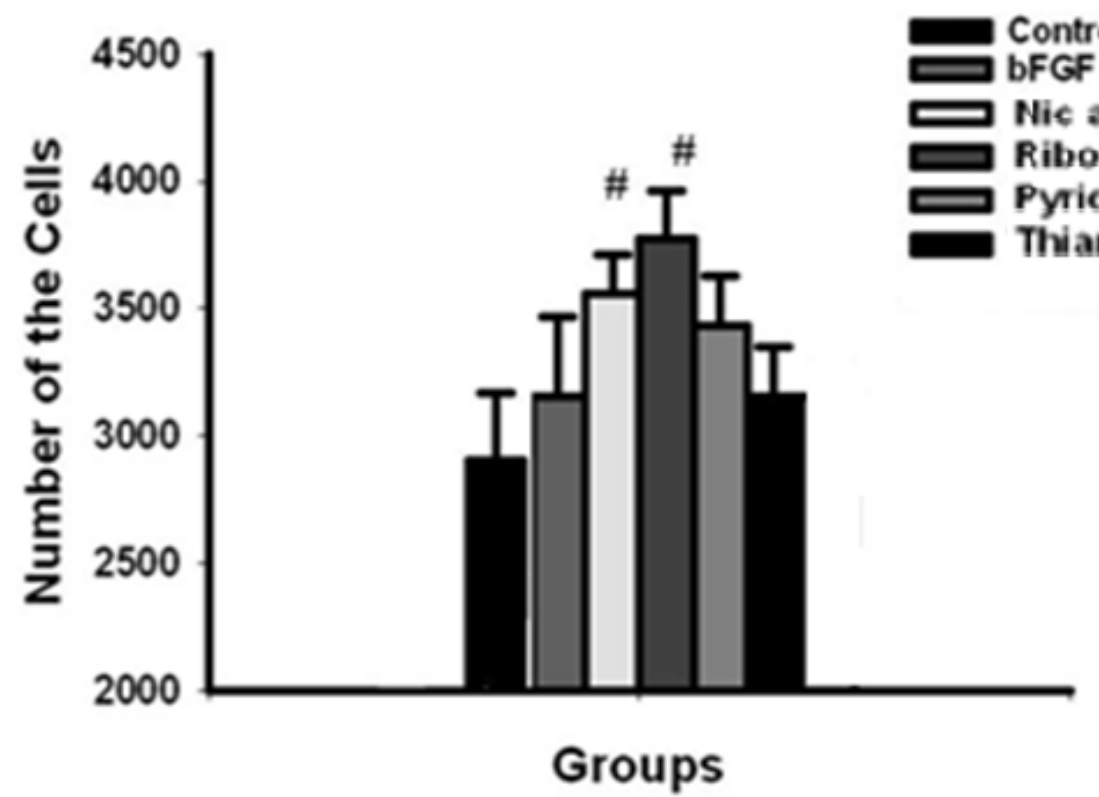

Polarizing microscope Bipolar PI (PZO,Warsaw, Poland; magnification 60x1.25x40) was used. The cells were grown on the glass in the Petry dishes ,covered with the Poly-L-Lysine. The Neurobasal/B27 or HBSS, containing bFGF, nicotinamide, riboflavin, pyridoxin, thiamine or low concentration of Vitamin B complex Was used as cell media.The number of the cells in the field was counted using Pixcavator program, allowing to calculate authomatically the size as well as the number of the cells.t-student test $\mathrm{t}$ was used to calculate the statistical significance of the results . $\mathrm{P}<$ 0.05 was considered significant

Figure 2.The influence of single components of Vitamin B complex on the growth and development of the human embryonic brain cells on day $12^{\text {th }}$
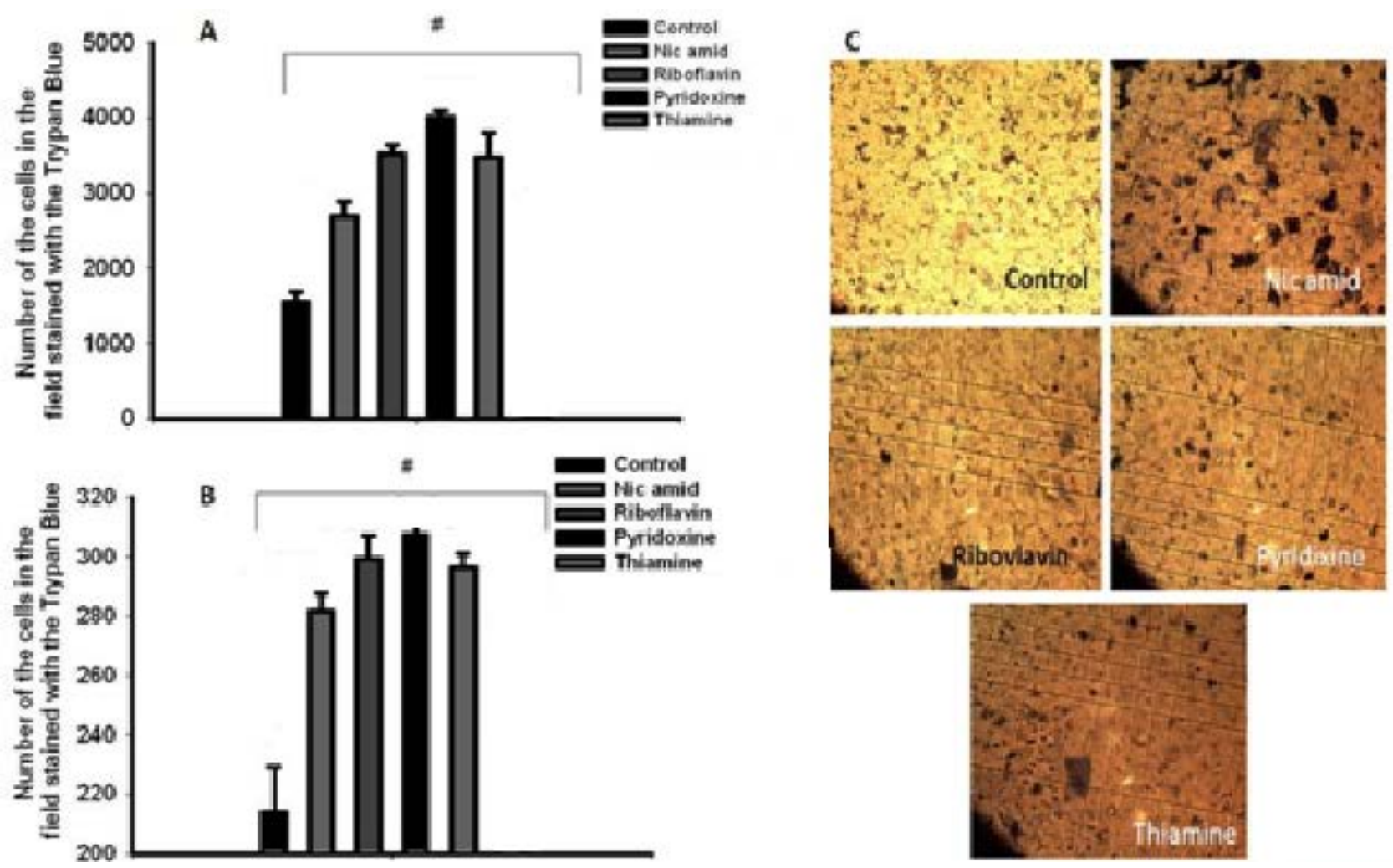
It has been used Polarizing microscope Bipolar PI (PZO,Warsaw,Poland; magnification 60x1.25x40). The cells were grown on the glass in the Petry dishes covered with the Poly-L-Lysine. The medium for cells served the Neurobasal/B27 or HBSS, containing nicotinamide, pyridoxin, thiamine or low concentration of vitamin B complex. The number of the cells in the field were counted by the utility of the Pixcavator programm, allowing authomatically calculatate the size as well as the number of the cells. Results were calculated utilizing SigmaStat 3.5. We have used one -wayANOVA for the comparison of the groups vs control or the groups between each other. The entire cells' fraction was removed from the wells, mixed for every group and $1 \mathrm{ul}$ of the fruction was pictured in the Hemocytometer. A. Trypan Blue staining of the fruction of the cells at day $\mathbf{4}^{\text {th }}$. P1 is the comparison of the results between the control and the all other groups. $(\mathbf{p}<\mathbf{0 . 0 0 1})$. $\mathbf{P}<\mathbf{0 . 0 5}$ was between nicotinamide and ribovlavin, $\mathbf{p}(<\mathbf{0 . 0 5})-$ nicotinamide and pyridoxine, $p(>0.05)$ - nicotinamide and thiamine, $p(>0.05)$ - riboflavin and pyridoxine, p7 ( >0.05)- riboflavin and thiamine, $p(>0.05)$ - pyridoxine and thiamine. B. Trypan Blue staining of the cells bodies on day $12^{\text {th }}$. P1 is the comparison of the results between the control and the all other groups. p<0.001. p ( $>0.05)$ - between nicotinamide and riboflavin, $\mathrm{p}(>0.05)$ - nicotinamide and pyridoxine, $\mathrm{p}(>0.05)$ - nicotinamide and thiamine, $\mathrm{p}(>0.05)$ - riboflavin and pyridoxine, $\mathrm{p}(>0.05)$ - riboflavin and thiamine, $\mathrm{p}(>0.05)$ - pyridoxine thiamine,C. The micropictures of the mixed fruction of the cells and cells' bodies.

Figure 3. Thrypan Blue staining of the human embryonic brain cells and cell bodies at day $12^{\text {th }}$
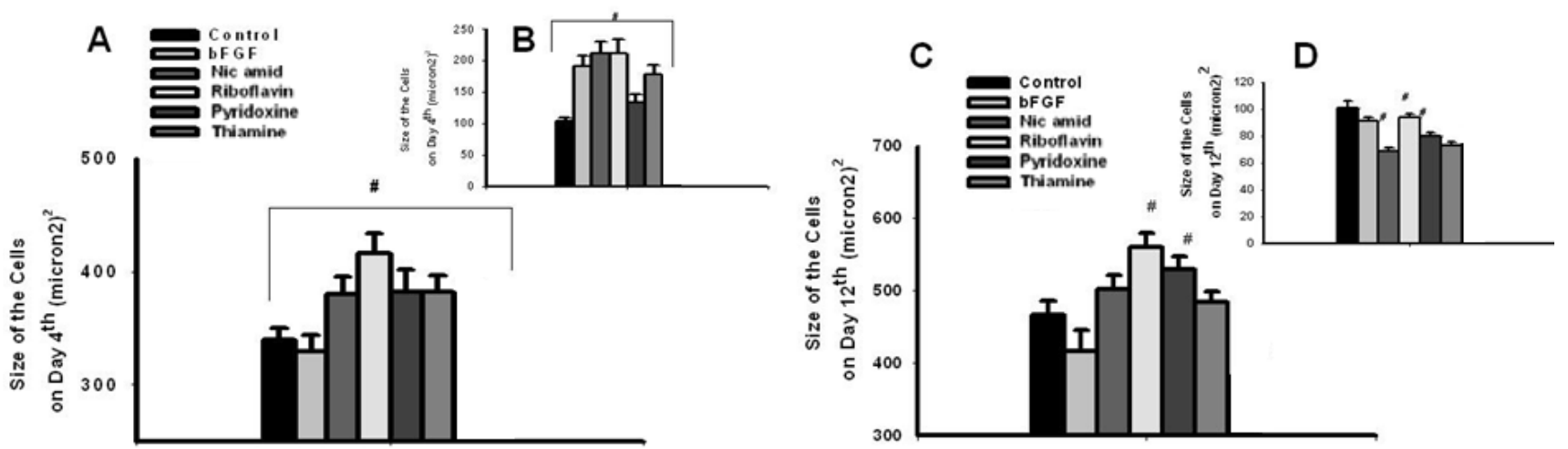

Polarizing microscope Bipolar PI (PZO,Warsaw,Poland; magnification 60x1.25x40) was used. The cells were grown on the glass in the Petry dishes ,covered with the Poly-L-Lysine. The Neurobasal/B27 or HBSS, containing nicotinamide,, pyridoxin, thiamine or low concentration of Vitamin B complex, served as media.The number of the cells in the field was countied using Pixcavator p as well as Imag Tool rogramm, allowing to calculate automatically the size as well as the number of the cells. Results were calculated by the utility of SigmaStat 3.5. We have used one -way-ANOVA for the comparison of the groups vs control or t-test for the coparison of two groups between each other. In the figures $\mathrm{A}$, B the $\mathrm{p}<0,05$ between control and the other groups. In figure C, D the difference between control and riboflavin, pyridoxine treated groups are statistically significant (C), as well as between nicotinamide, riboflavin and pyridoxine treated groups of the small cells fruction $(\mathrm{p}<0,05, \mathrm{D})$

Figure 4. Measurement of the cells' and embryonic bodies' sizes on the day $4^{\text {th }}(\mathrm{A}, \mathrm{B})$ and $12^{\text {th }}(\mathrm{C}, \mathrm{D})$

A

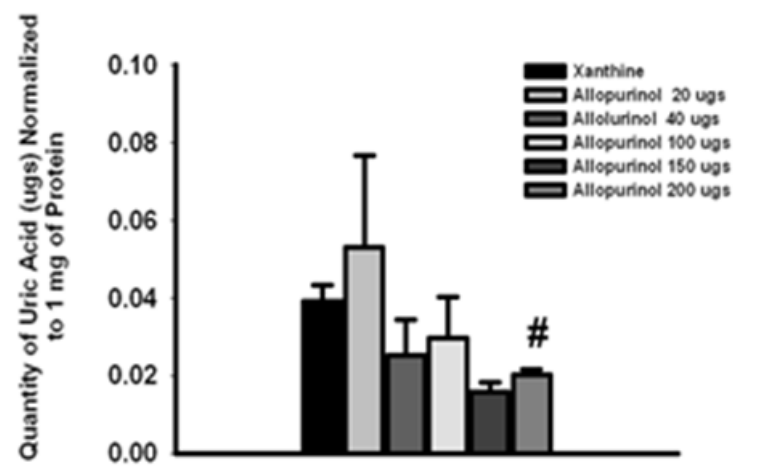

B

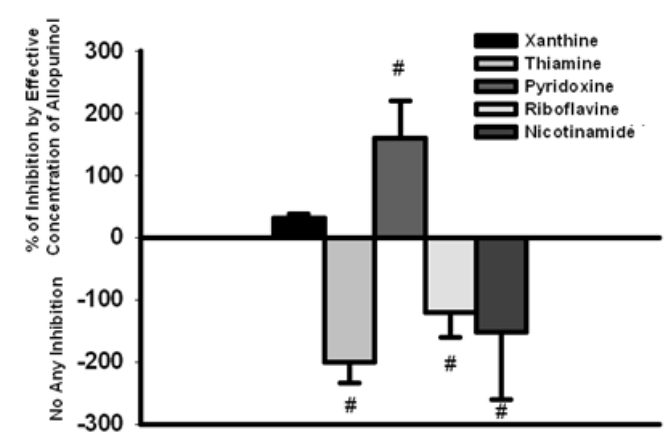

A. Inhibtion of Uric acid formation in the human brain derived (E90) cells by the utility of allopurinol. The first black column represents the ugs of Uric acid ,formed in the presence of the substrate of XO - xanthine. Further application of allopurinol in the concentration equal to 0,0024uM, 0,0048uM; 0,0097 uM, 0,018 uM, 0,036 uM was in the concentration dependent manner decreasing the total quantity of Uric acid, normalized to 1 mg of the protein. Statistically significant difference was observed during the copmarison of the control group vs allopurinol, applied in the concentration of $0,036 \mathrm{uM}(\mathrm{p}<0,05)$.

B. Persentile of inhibition of Uric acid formation in the pressence of Vitamin B subcomponents. The first black bar sows the persentile of uric acid formation during the 40 minutes of incubation in the presence of xanthine and allopurinol (31, 5 $\pm 6,9)$. The second gray bar situated above the X-axis indicates the persentile of pyridoxine dependent, initiated by allopurinol inhibition of XO, which is compared and calculated based on the quantity of uric acid formated in the presence of pyridoxine. The persentiles with the sign "_" show no inhibition of uric acid formation, because of the presence in the samples riboflavin, thiamine, nicotinamide. The all comparisons between control and experimental groups are statistically significant and $\mathrm{p}<0,05$

Figure 5. Assessment of XO activity by the estimation of Uric acid formation in the presence of allopurinol and Vitamin B complex subcomponents 


\subsection{Measurement of the Cells' and Embryonic Bodies' Sizes on the $4^{\text {th }}$ and $12^{\text {th }}$ Days}

Using Pixcavator 2.1 program, we have measured the size of the cells in the cell culture and evaluated the sizes of the embryonic bodies. From the Figure 4 it is clear that the most prominent compound, which induced the development of the small cells fraction and their enlargement in the statistically significant way in comparison with the control group $(103.84 \pm 5.68)$ were

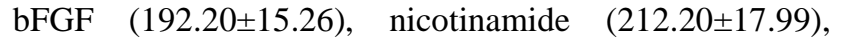
$\mathrm{p}(212.72 \pm 21.89)$, as well as Pyridoxine (133.75 \pm 13.53$)$ and thiamine $(177.89 \pm 16.14)$. Large fraction of the cells or cells' bodies were more sensitive to the riboflavin $(416.11 \pm 18.18)$ influence on day $4^{\text {th }}$ in comparison with the control group $(339.72 \pm 9.92, \mathrm{p}<0.01)$. Moreover, the entire experimental group induced the enlargement of the cells in accordance to the calculation by One-Way-Anova in the comparison with the control groups in the statistically significant way (FGF 329.13 \pm 14.15 , nicotinamidee-380.33 \pm 14.90 , pyridoxine $-382.13 \pm 19.26$, thiamine-382.51 \pm 13.68$)$.

On day 12th large fraction of the cells was in the statistically significant way sensitive to the influence of the riboflavine and pyridoxine vs control (572.67 \pm 17.91 , $512.50 \pm 17.64$ vs $416.64 \pm 28.83, \mathrm{p}<0,05)$ in contrast to the other groups (bFGF 496.09 \pm 21.16 , nicotinamide 501.86 \pm 19.07 , thiamine 484.69 \pm 14.82 ). The most important and interesting observation for us was the increase in the average size of the large fraction from 400 (day 4th) to 500 (day 12th). The size of the small cells' fraction in the experimental groups was lower in comparison even with the control group (90.90 \pm 2.86$)$, bFGF (100.92 $\pm 4.95, \quad \mathrm{p}<0,05$ vs control), nicotinamide (68.86 $\pm 2.65, \mathrm{p}<0,05$ vs control), riboflavin (93.28 \pm 2.65 , $\mathrm{p}<0,05$ vs control), pyridoxine (80.06 \pm 2.58$)$, thiamine (72.96 \pm 2.43$)$.

\subsection{Assesment of XO Activity by the Estimation of Uric Acid Formation in the Presence of Vitamin B Complex Subcomponents}

Taking into the consideration the fact that the most of the components of Vitamin B complex separately has the impact on the prevention of reactive oxygen spices formation, influence on the cells growth and maturation, we have evaluated the activity of $\mathrm{XO}$ in the human brain derived cells in the presence of above mentioned subcomponents $[8,16]$. In the first set of the experiments we have evaluated the XO activity in the presence of applied several concentrations of allopurinol 0,36ug; 0,72ug; 1,44ug; 2,7ug; 5,4ug. $\quad\left(0.0393 \pm 3.9277^{\mathrm{e}-3}\right.$, $0.0531 \pm 0.0236, \quad 0.0252 \pm 9.1646^{\mathrm{e}-3}, \quad 0.0296 \pm 0.0105$, $0.0157 \pm 2.6185^{\mathrm{e}-3}, 0.0202 \pm 1.3092^{\mathrm{e}-3}, \mathrm{p}<0,05$ between the control, and 5,4 ug of allopurinol). As it is clear from the Figure 5 A. allopurinol was inhibiting the activity of $\mathrm{XO}$ in the concentration dependent manner.

During the next set of the experiments, we have evaluated the influence of the Vitamin B complex subcomponents on the activity of XO. In the Figure $5 \mathrm{~B}$ it is presented the \% of the $\mathrm{XO}$ inhibition in the presence of the subcomponents of $\mathrm{B}$ complex. The calculations are based on the quantity of detected uric acid normalized to 1 $\mathrm{mg} f$ the protein. Percentile of inhibition/ non inhibition of all subcomponents of Vitamin B complex, thiamine, pyridoxine, riboflavin, nicotinamide, (-201,39 $\pm 32,76$, $160,00 \pm 60,00,-120,91 \pm 39,091,-152,73 \pm 107,27$, resp.) were in statistically significant way different from the control sample $(31,0343 \pm 6,9222, \mathrm{p}<0,05)$, which is the percentile of inhibition of $\mathrm{XO}$ in the presence of allopurinol and absence of subcomponents.

In our previous experiments we have shown that Vitamin B complex initiates the cells' growth and maturation. Results presented in this article indicate that the all components of Vitamin B complex are responsible for these functions. During the early period of the growth, important components responsible for these functions were thiamine and pyridoxine, riboflavin as well as nicotinamide. However, the most vivid difference might be detected in the groups treated with the pyridoxine and thiamine.

The size of the embryonic bodies, as well as smaller cells, was sensitive to the all components of Vitamin B subcomponents. At day $12^{\text {th }}$ the size of the small cells' fraction treated with the subcomponets of Vitamin B complex was smaller in comparison with the control groups. Instead, the average size of the embryonic bodies in comparison with day $4^{\text {th }}$ increased by $20 \%$ in the groups treated with the subcomponents, indicating on the fact that small cells were gathering together and forming larger embryonic bodies.

Our previous results [2] indicated that the early inhibition of the $\mathrm{XO}$ in the human brain derived cell culture utilizing allopurinol were initiating the increase in the number of the cells in comparison with the later stage of inhibition.

In comparison with the all other subcomponents of Vitamin B complex, only in pyridoxine containing samples XO activity was specifically inhibited by allopurinol. Moreover, pyridoxine by itself was inhibiting formation of uric acid. In all the other samples XO activity wasn't inhibited with no any concentrations of allpurinol, probably due to the initiation of the alternative ways of uric acid formation.

Similarly to allopurinol pyridoxine treatment during the early stages was initiating the increase in the number of the cells, whereas in the late stages that process was suppressed. During the late stages the most effective components were riboflavin and nicotinamide.

Also, we would like to mention that allopurinol is the classical drug for the treatment of chronic gout [12]. Our results indicates that the effectiveness of this compound might be diminished in case of simultaneous treatment of the patients along with the allopurinol, as well as also with riboflavin, nicotinamide and thiamine, because of possible stimulation of the other biochemical pathways responsible for the formation of Uric acid.

We have concluded that pyridoxine might interact with $\mathrm{XO}$ and regulate its activity. All components of Vitamin B complex are able to initiate cells development and growth; however, they are supposed to be selectively utilized in 
time dependent manner to guarantee the highest effectiveness of the cell development, maturation and proliferation.

\section{Acknowledgment}

This work was made possible by a research grant from the Armenian National Science and Education Fund (ANSEF) based in New York, USA. We are thankful to S. Alaverdyan, who was helpful with her work related with the cells' size measurement, cells' count by the utility of Pixcavator and Image Tool programs.

\section{References}

[1] Castro, G.D., Costantini, M.H. and Castro, J.A., Rat ventral prostate xanthine oxidase-mediated metabolism of acetaldehyde to acetyl radical, Hum Exp Toxicol, 28(4) (2009 ) 203-8.

[2] Danielyan, K.E. and Kevorkian, G.A., Xanthine oxidase activity regulates human embryonic brain cells growth, Biopolym. Cell, 27(5) (2011) 350-353.

[3] Geng, M.-Y., Saito, H. and Katsuki, H., Effects of vitamin B6 and its related compounds on survival of cultured brain neurons, Neuroscience Research, 24 (1995) 61-65.

[4] Geng, M.Y., Saito, H. and Katsuki, H., The effects of thiamine and oxythiamine on the survival of cultured brain neurons, Jpn J Pharmacol, 68 (1995) 349-52.

[5] Geng, M.Y., Saito, H. and Katsuki, H., Effects of vitamin B6 and its related compounds on survival of cultured brain neurons, Neurosci Res, 24 (1995) 61-5.

[6] Hille, R. and Nishino, T., Flavoprotein structure and mechanism. 4. Xanthine oxidase and xanthine dehydrogenase, FASEB J, 9 (1995) 995-1003.

[7] Kruger, N.J., The Bradford method for protein quantitation, Methods Mol Biol, 32 (1994) 9-15.
[8] Lucius, R., Mentlein, R. and Sievers, J., Riboflavin-mediated axonal degeneration of postnatal retinal ganglion cells in vitro is related to the formation of free radicals, Free Radic Biol Med, 24(5) (1998) 798-808.

[9] Mattson, M.P. and Ruchlik, B., Cell culture of cryopreserved human fetal cerebral cortical and hippocampal neurons: neuronal development and resposes to trophic factors, Brain Research, 552 (1990) 2004-212.

[10] Mukherjee, S.K., Klaidman, L.K., Yasharel, R. and Adams, J.D.J., Increased brain NAD prevents neuronal apoptosis in vivo, Eur J Pharmacol, 330 (1997) 27-34.

[11] Olson, J.S., Ballou, D.P., Palmer, G. and Massey, V., The mechanism of action of xanthine oxidase, J. Biol. Chem, 249 (1974) 4363-4382.

[12] Pacher, P., Nivorozhkin, A. and Szabó, C., Therapeutic Effects of Xanthine Oxidase Inhibitors: Renaissance Half a Century after the Discovery of Allopurinol, Pharmacological Reviews, 58 (1) (2006) 87-114.

[13] Patt, A., Harken, A.H., Burton, L.K., Rodell, T.C., Piermattei, D., Schorr, W.J., Parker, N.B., Berger, E.M., Horesh, I.R. and Terada, L.S., Xanthine oxidase-derived hydrogen peroxide contributes to ischemia reperfusion-induced edema in gerbil brains, J Clin Investig, 81 (1988) 1556-1562.

[14] Rahman, A., Katzive, L., Stanley, K. and Henshaw, S.K., A Global Review of Laws on Induced Abortion, International Family Planning Perspectives1985-1997, 24 (1998).

[15] Sanfeliu, C., Wright, J.M. and Kim, S.U., Neurotoxicity of isoniazid and its metabolites in cultures of mouse dorsal root ganglion neurons and hybrid neuronal cell line, Neurotoxicology, 20(6) (1999) 935-44.

[16] Sheline, C.T., Zhou, Y. and Bai, S., Light-induced photoreceptor and RPE degeneration involve zinc toxicity and are attenuated by pyruvate, nicotinamide, or cyclic light, Mol Vis, 16 (2010) 263952.

[17] Tennant, J.R., Evaluation of the trypan blue techni2que for determination of cell viability, Transplantation, 2 (1964) 685-94.

[18] Theron, A., Anderson, A., Grabow, G. and Meiring, J.L., In vitro and in vivo stimulation of neutrophil migration and lymphocyte transformation by thiamine related to inhibition of the peroxidase/H202/halide system, Clin. exp. Immunol, 44 (1981) 295-303. 